\title{
Expression of CD80 and CD86 on B cells during coxsackievirus B3-induced acute myocarditis
}

\author{
YANLAN HUANG, BIN WEI, XINGCUI GAO, YAN DENG, WEIFENG WU
}

Department of Cardiology, First Affiliated Hospital of Guangxi Medical University, Guangxi, China

\begin{abstract}
Introduction: The pathogenesis of viral myocarditis (VMC) is unclear, but many studies have shown that VMC is associated with an excessive immune response. CD80 and CD86 are important costimulatory molecules that play a critical role in autoimmunity. However, whether CD80+/CD86+ B cells participate in the pathogenesis of acute VMC is unknown.

Material and methods: Male C57BL/6 mice were infected by intraperitoneal injection with coxsackievirus B3 (CVB3) to establish a VMC model. Control mice were administered phosphate-buffered saline intraperitoneally. At one week and two weeks post injection, histopathological changes in heart tissue were assessed with haematoxylin and eosin staining. The frequency of splenic CD80+/CD86+ $B$ cells was measured with flow cytometry.

Results: The frequency of CD80+B cells was significantly increased in VMC, while the frequency of $C D 86+B$ cells was significantly decreased. Furthermore, the frequency of CD80+B cells related to the severity of VMC.

Conclusions: These data show that CD80+/CD86+B cells are involved in the pathogenesis of VMC, with $C D 80+B$ cells being more important than $C D 86+B$ cells.
\end{abstract}

Key words: B cells, CD80, CD86, viral myocarditis.

(Centr Eur J Immunol 2019; 44 (4): 364-369)

\section{Introduction}

Viral myocarditis (VMC) is the primary cause of sudden death and heart failure in adolescents $[1,2]$. However, no effective treatment is available because the pathogenesis of VMC is unclear. It is widely believed that pathogenesis is chiefly due to an excessive immune response to viral infection [3], in which myocardial damage during acute VMC is due to T lymphocytes. T-lymphocyte activation requires not only $\mathrm{T}$-cell receptor recognition of the major histocompatibility complex (MHC)-viral peptide complex on antigen-presenting cells (APCs), but also T-cell CD28 interaction with B7, on APCs [4].

CD80 and CD86 belong to the B7 family of costimulatory molecules and play a key role in T-cell activation and regulation [5]. Studies have shown that activated B cells, $\mathrm{T}$ cells, and dendritic cells express high levels of CD80 and CD86 [6-8], whereas resting B cells, T cells, and dendritic cells do not express high levels of these molecules. Seko et al. $[9,10]$ found that CD80 and CD86 were expressed on ventricular tissues of mice and humans with acute myocarditis as well as in humans with dilated cardiomyopathy. Although those findings indicated that CD80 and CD86 could play an important role in myocardial injury, the stud- ies did not identify the cellular source for CD80 and CD86. Others have shown CD80 and CD86 to be expressed on disease-involved B cells in multiple sclerosis [11], rheumatoid arthritis [12], and asthma [13]. In particular, O'Neill et al. [14] demonstrated CD80+/CD86+ B cells to play a crucial role in the development of arthritis. However, little is known about the potential role of CD80+/CD86+ $\mathrm{B}$ cells in VMC. In this study the expression of CD80 and CD86 on B cells was investigated in a coxsackievirus B3 (CVB3)-induced acute VMC murine model, to assess possible clinical treatment modalities for VMC.

\section{Material and methods}

\section{Mice}

Specific pathogen-free, male, four-week-old C57BL/6 mice were obtained from the Laboratory Animal Centre of Foshan (Guangdong Province, China). All animals were housed in a pathogen-free mouse room in the Guangxi Medical University Laboratory Animal Centre (Nanning, China). Experiments were implemented according to protocols approved by Guangxi Medical University Animal Ethics Committee.

Correspondence: Weifeng Wu MD, PhD, Department of Cardiology, First Affiliated Hospital of Guangxi Medical University,

Nanning 530021, Guangxi, China, e-mail: wucna65@163.com

Submitted: 26.09.2017; Accepted: 21.09.2018 


\section{Virus}

CVB3 (Nancy strain) was passaged in HEp-2 cells. Virus titres were assessed with plaque-forming unit (PFU) assays at $1 \times 107$. C57BL/ 6 mice were infected intraperitoneally with $105 \mathrm{PFU}$ of CVB in $0.1 \mathrm{ml}$ phosphate-buffered saline (PBS) to induce the acute VMC model.

\section{Myocarditis model}

A total of 36 male C57BL/6 mice were randomly divided into two groups: the VMC group $(n=20,10$ mice/ group) and the control group ( $n=16,8$ mice/group). Each group was divided into two subgroups: one week (1w) and two weeks $(2 \mathrm{w})$. Mice intraperitoneally injected with $0.1 \mathrm{ml}$ of PBS containing $\sim 1 \times 105$ PFU of CVB3 were the $\mathrm{VMC}$ group. Mice treated intraperitoneally with $0.1 \mathrm{ml}$ PBS were the control group. Surviving animals of each group were euthanised at $1 \mathrm{w}$ and $2 \mathrm{w}$ after injection. Hearts as well as spleens were removed aseptically, immediately after euthanasia, for further assessment.

\section{Histopathology}

The heart was cut longitudinally, fixed in $4 \%$ paraformaldehyde, and embedded in paraffin. Tissues were cut into $5 \mu \mathrm{m}$-thick sections and processed by haematoxylin and eosin (H \& E) staining. Histopathological changes were observed under light microscopy (magnification 400x). Myocardial pathology scores were determined by two independent investigators according to a semi-quantitative scale [15]: grade 0 , no cardiac inflammation; grade 1 , $<25 \%$; grade 2 , between $25 \%$ and $50 \%$; grade 3 , between $50 \%$ and $75 \%$; and grade $4,>75 \%$.

\section{Flow cytometry}

Spleens were harvested aseptically and dissected from surviving mice. Cell suspensions were prepared by passage through nylon mesh, and red cells were removed with Lysing Buffer (BD). Splenocytes were resuspended as single cells in PBS and assessed with flow cytometry using the following antibodies: FITC-CD19, PE-CD86, APC-CD80, which were all purchased from eBioscience. The cells were stained with these antibodies for $30 \mathrm{~min}$ at $4^{\circ} \mathrm{C}$ in the dark. Isotype control antibodies (eBioscience) were used to control for non-specific antibody binding to the cells. Cells were then washed, resuspended in PBS, and analysed with a BD FACSCanto II flow cytometer. Data were analysed with FlowJo7.6 software.

\section{Statistics}

Data are presented as mean \pm SD. Student's t-test was used for two-group comparisons. Correlations were made by Spearman's test. All statistical analysis was completed with SPSS17.0. Statistical significance was set at $p<0.05$.

\section{Results \\ VMC severity}

There were nine survivors in the $1 \mathrm{w}$ VMC subgroup and in the $2 \mathrm{w}$ VMC subgroup, respectively. No deaths were observed in the control group. For the $1 \mathrm{w}$ VMC subgroup there was extensive heart inflammatory cell infiltration and necrosis. By contrast, there was little less inflammatory cell infiltration and necrosis in the hearts of the $2 \mathrm{w}$ VMC subgroup. No inflammatory cell infiltration or necrosis was observed in the hearts of the control group (Fig. 1A). The myocardial pathology scores for the VMC groups are summarised in Figure 1B.

\section{CD80+ B cells, not CD86+ B cells, were elevated in $\mathrm{VMC}$}

To quantify the surface expression of CD80 and CD86 on B cells in the acute VMC mice, the frequency of CD80+/CD86+ B cells was measured with flow cytometry (Fig. 2A, B). The frequency of CD80+ B cells was significantly increased in the VMC group compared to the control group at $1 \mathrm{w}$ and $2 \mathrm{w}(7.62 \pm 0.47 \%$ vs. $4.29 \pm 0.52 \%$; $5.94 \pm 0.60 \%$ vs. $4.11 \% \pm 0.36 \%$, respectively, $p<0.01$, Fig. 2C). In contrast, the frequency of CD86+ B cells was significantly decreased in the VMC group when compared to the control group at $1 \mathrm{w}$ and $2 \mathrm{w}(1.57 \pm 0.44 \%$ vs. 3.85 $\pm 0.59 \% ; 1.45 \pm 0.42 \%$ vs. $3.63 \pm 0.45 \%$, respectively, $p<0.01$, Fig. 2D). In addition, the frequency of CD80+ $\mathrm{B}$ cells was higher at $1 \mathrm{w}$ in the VMC subgroup than in the 2w VMC subgroup $(p<0.01)$. There was no statistical difference in CD86+ B cells when the $1 \mathrm{w}$ VMC subgroup was compared to the $2 \mathrm{w}$ VMC subgroup $(p>0.05)$.

\section{CD80+ B cell expression was related to VMC severity}

To determine whether CD80+/CD86+B cells are associated with the severity of inflammation in VMC, flow cytometry results were related to heart tissue pathology scores. The frequency of CD80+ B cells was positively related to the severity of $\mathrm{VMC}$ heart pathology scores $(p<0.01)$ (Fig. 3), while the frequency of CD86+ B cells was not (data are not shown).

\section{Discussion}

In the pathogenesis of VMC, a number of studies have shown that $\mathrm{T}$ cells play an important role, while $\mathrm{B}$ cells do not. In this study, CD80+ B cells increased and CD86+ $\mathrm{B}$ cells decreased during VMC. CD80+ B cells were positively related to disease severity.

CD80 and CD86 are important costimulatory molecules. Although CD80 and CD86 provide synergistic stimulation for T-cell activation, CD80 promotes the differenti- 

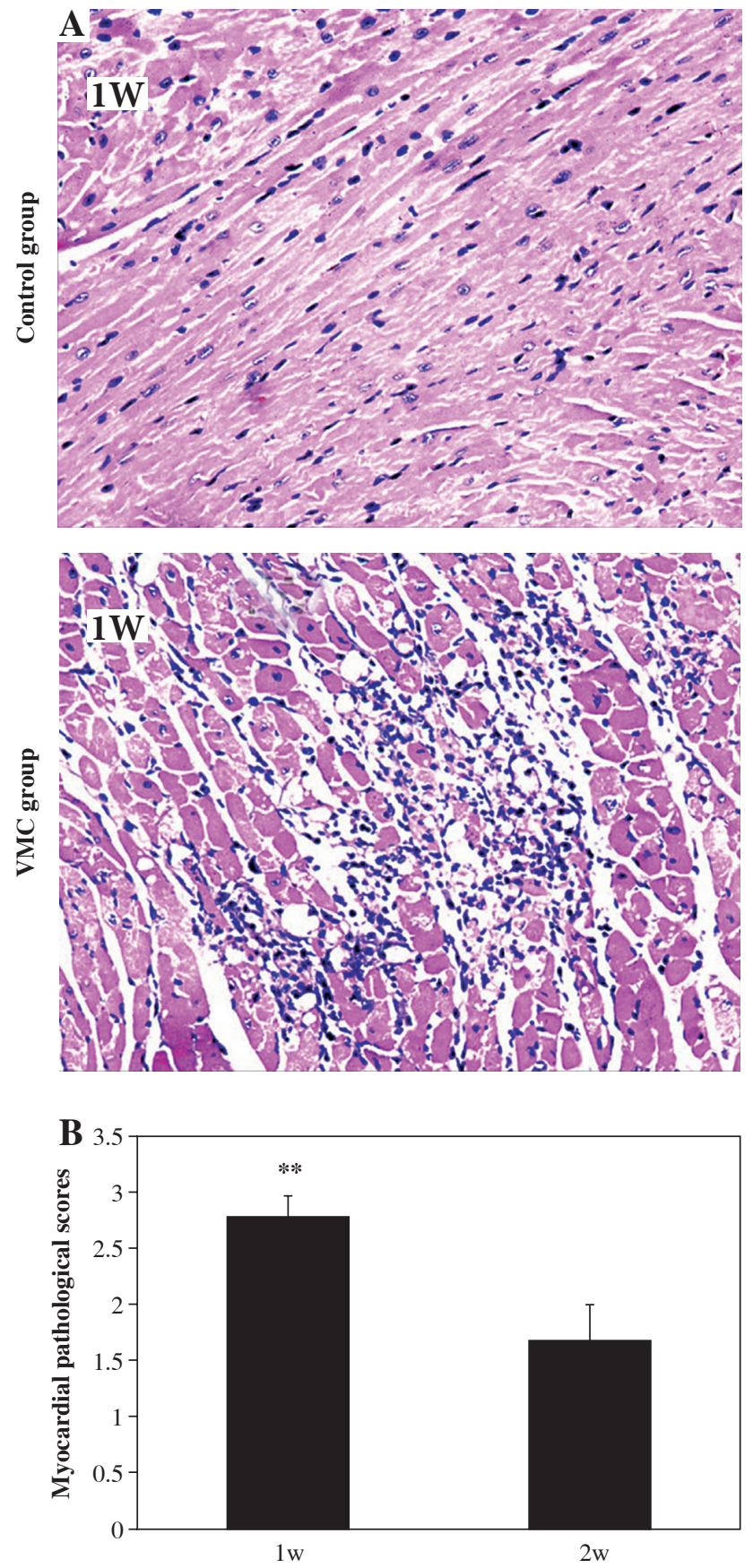

ation of T cells into Th1 cells, while CD86 mainly induces Th2-mediated humoural immunity and immunoglobulin production. These costimulatory molecules play an important role in T-cell activation and immune upregulation during autoimmunity [16], with an imbalance of CD80 and CD86 associated with the occurrence of autoimmune diseases [17].

CD80 and CD86 play important roles in the immune system and cannot be replaced by other molecules [18, 19]. In animal models, CD80 is associated with pro-inflamma-
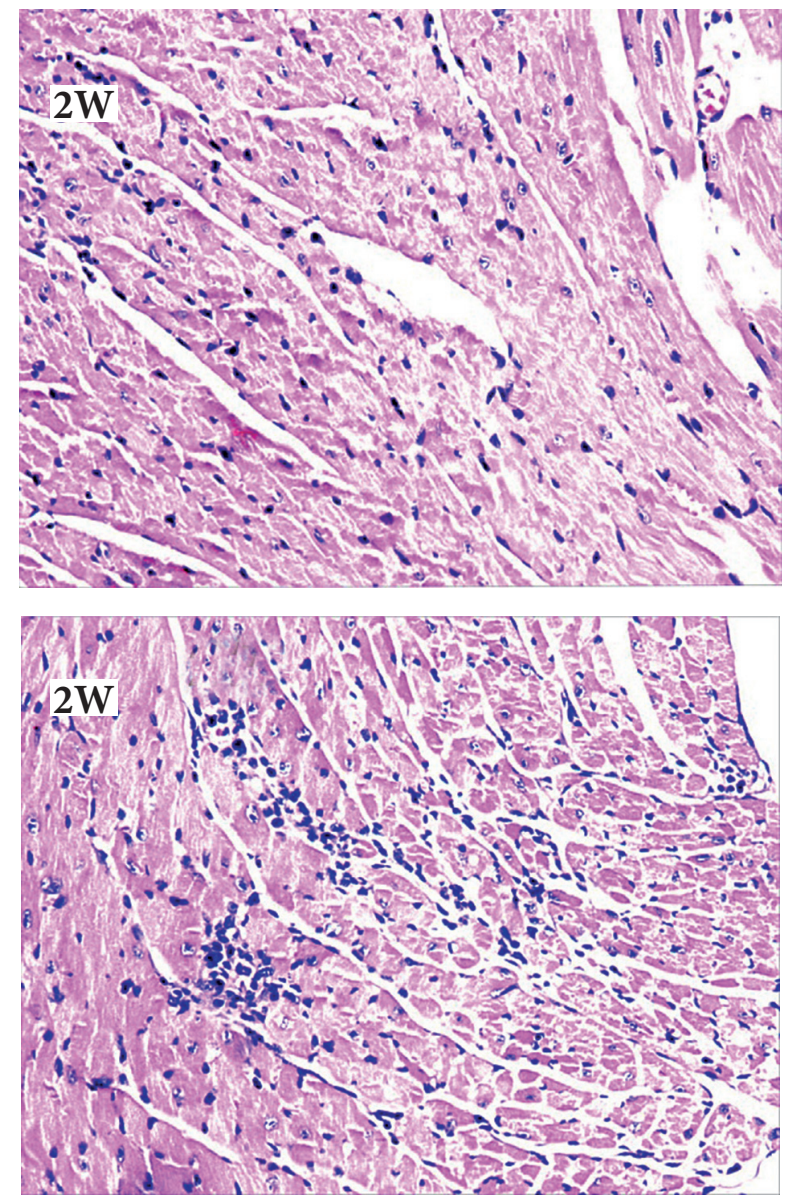

Fig. 1. A) Representative myocardial histopathologic images in control and VMC animals ( $\mathrm{H} \& \mathrm{E}$, original magnification $\times 400$ ). B) The results of myocardial pathology scores in the VMC group, $n=9$ mice/subgroup, \#\# $p<0.01$ vs. 2 w VMC subgroup

tory effects, whereas CD86 is associated with anti-inflammatory effects [20]. Kuchroo et al. [21] demonstrated that treatment with anti-CD80 antibody reduced the incidence of experimental allergic encephalomyelitis (EAE), whereas administration of anti-CD86 antibody increased EAE disease severity. Furthermore, CD80 and CD86 function differently in different tissues [22, 23] and cells. Nolan et al. [24] demonstrated that during sepsis CD80 expression was upregulated, while CD86 expression was downregulated with differing expression of CD80/CD86 in different tissues. 
The aforementioned studies analysed all APCs. More recently, CD80/CD86 expression on B cells was shown to drive autoimmune pathogenesis. Soraya et al. [11] demonstrated CD80 and CD86 on B cells to be elevated in all human T-cell lymphotropic virus 1 infected individuals. Sellebjerg et al. [11] demonstrated that CD80 expression on B cells was increased in the cerebrospinal fluid of multiple sclerosis patients with increased CD80+B cells, corresponding to increased disease severity. In patients with rheumatoid arthritis, Leng et al. [25] showed that CD86 expression was significantly decreased, while CD80 expression did not differ on peripheral blood B cells. With regard to VMC, CD80/CD86 expression on $\mathrm{B}$ cells has not been previously reported.

This study showed that CD80 expression on B cells was significantly elevated while CD86+ B cell expression
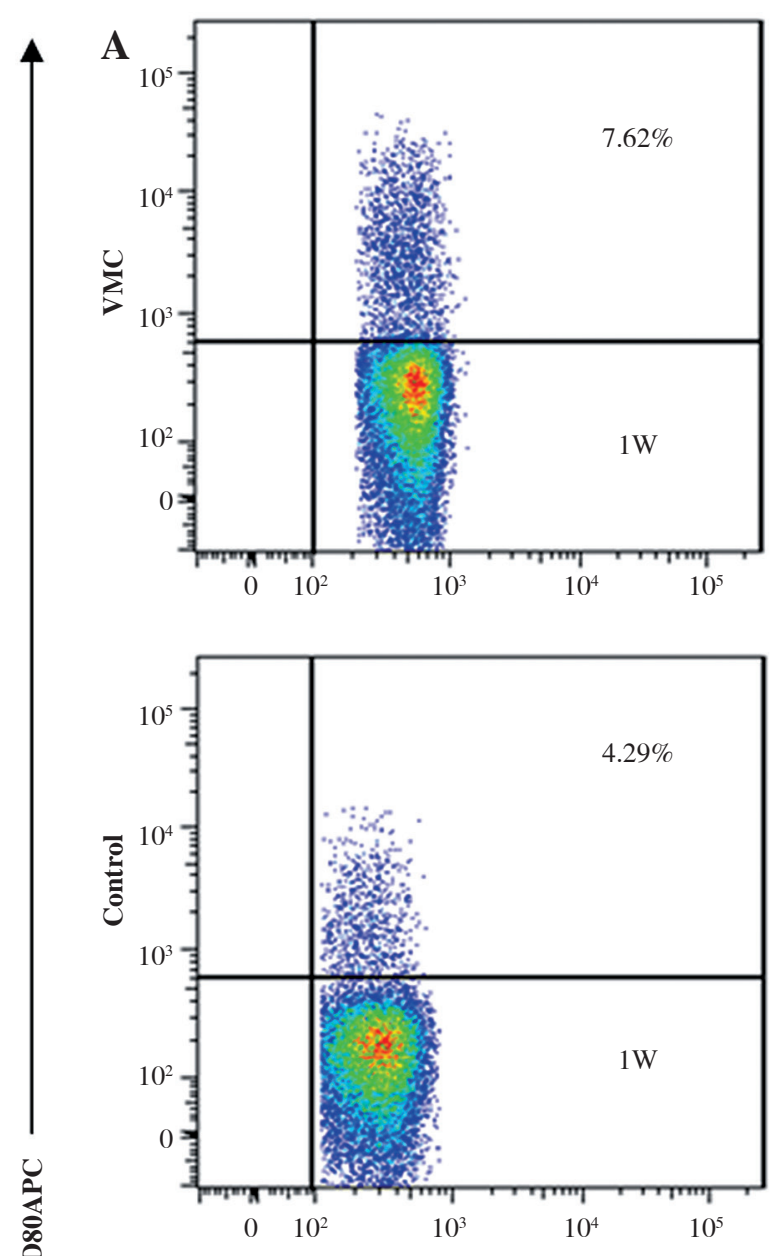

was significantly decreased in acute VMC. In addition, we found that elevated CD80+B cells but not CD86+ B cells were associated with the severity of inflammation during VMC. These results suggest that CD80 is an important B-cell costimulatory molecule and that pro-inflammatory CD80+ B cells are more important than the anti-inflammatory CD86+B cells during acute VMC. These results, similarly to previous studies in other diseases, demonstrate differential B-cell expression of CD80/CD86 in acute VMC.

\section{Conclusions}

Our results, for the first time, demonstrate that CD80+/ $\mathrm{CD} 86+\mathrm{B}$ cells play an important role in the pathogenesis
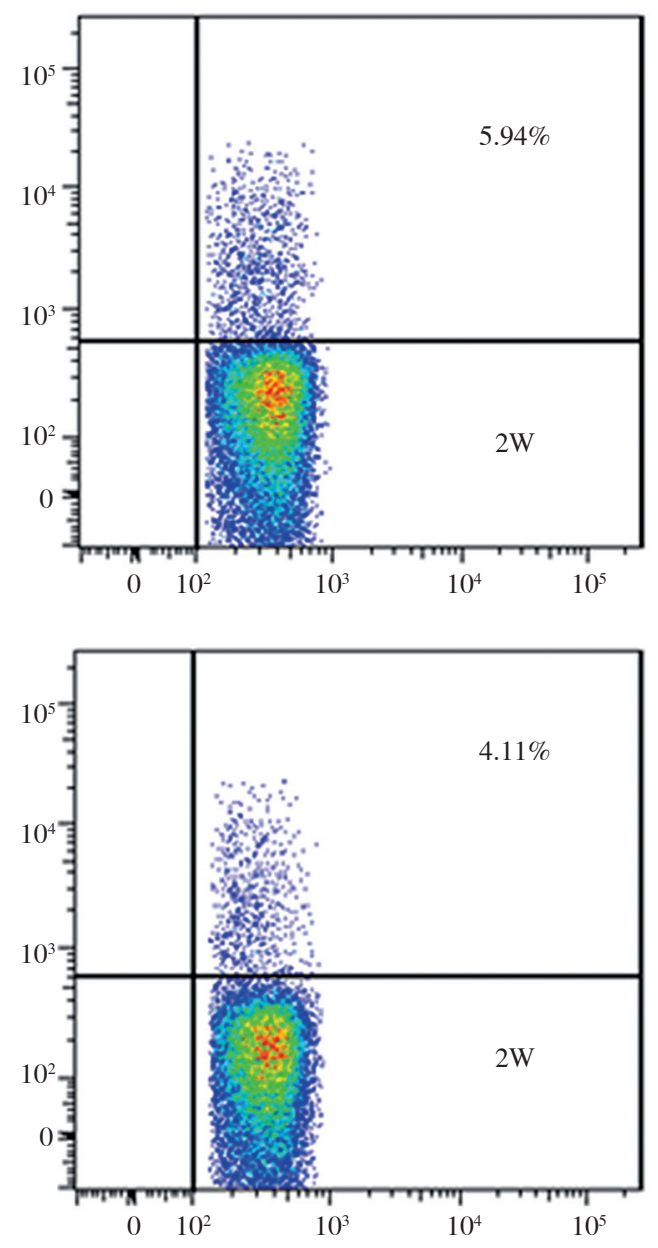

\section{CD19 FITC}

Fig. 2. CD80+B cells, not CD86+B cells, were increased in VMC. A) Representative pictures from VMC and control mice for the frequency of CD80+ B cells, B) representative pictures from VMC and control mice for the frequencies of CD86+ B cells, C) the results of CD80+ B cell statistical analysis, D) the results of CD86+ cell statistical analysis. ${ }^{* *} p<0.01$ vs. control group $(n=8)$. ${ }^{\#} p<0.01$ vs. $2 \mathrm{w}$ VMC subgroup $(n=9)$. Data are presented as mean $\pm \mathrm{SD}$ 

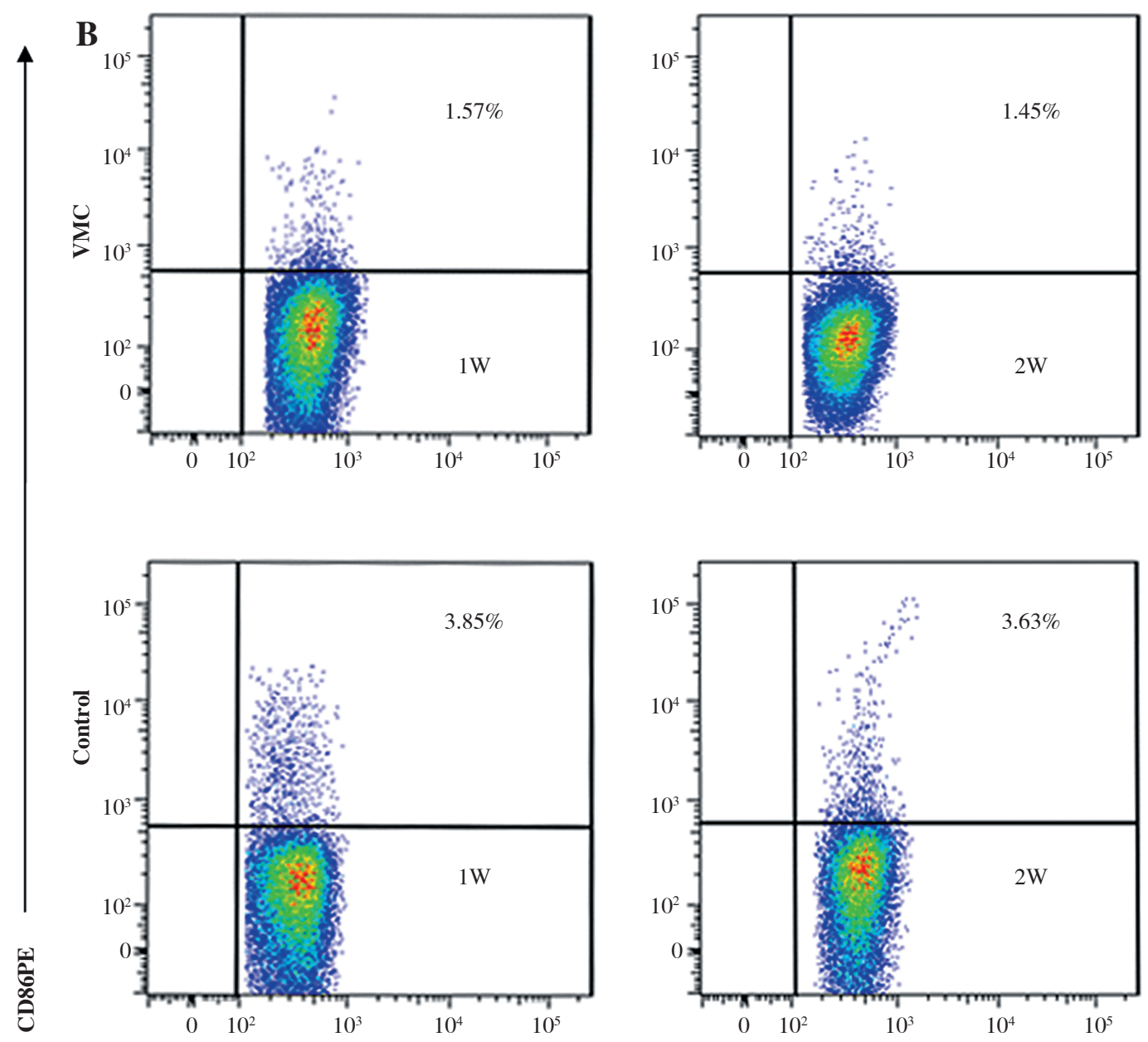

CD19 FITC
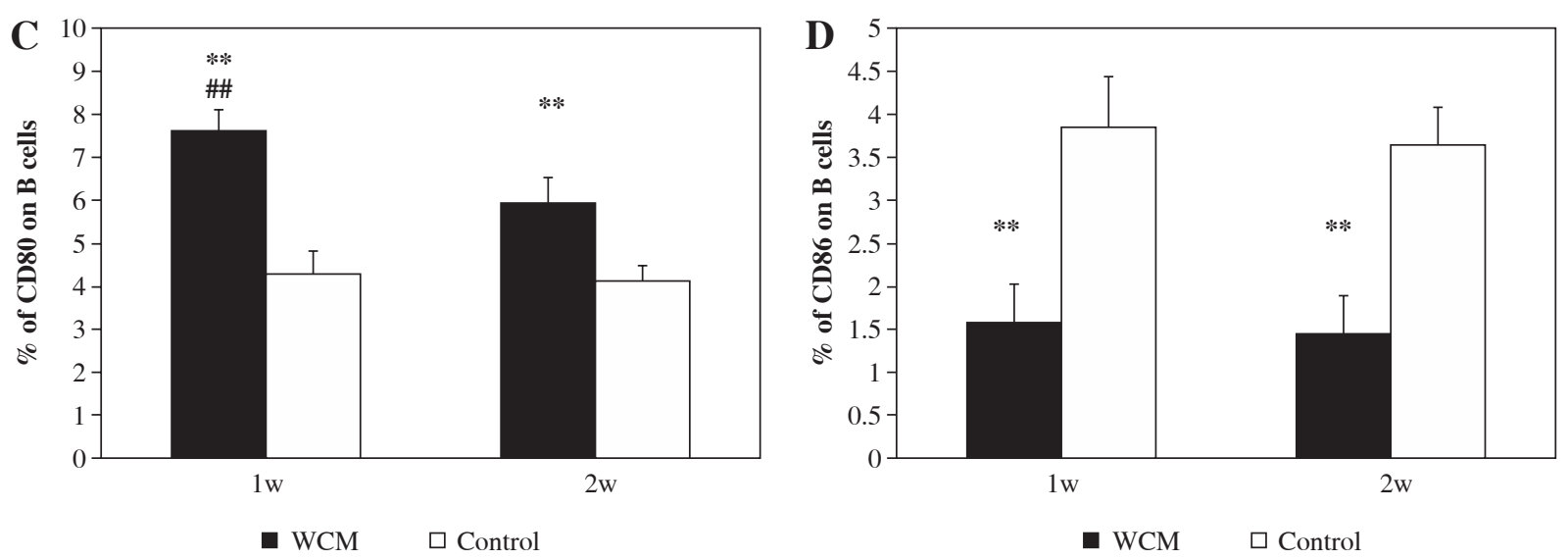

Fig. 2. Cont. 


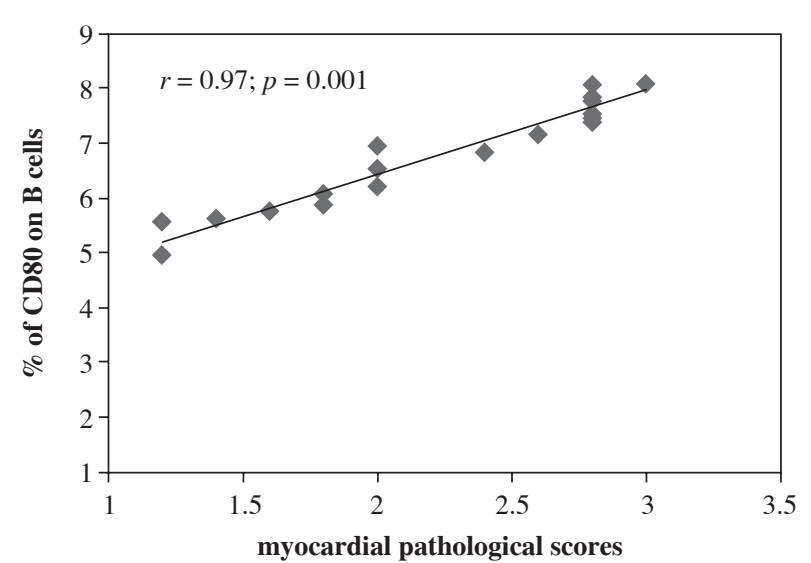

Fig. 3. The correlation of CD80+ B cell frequency with pathology scores of heart tissues in VMC mice; $r=0.97$, $p=0.001(n=18)$

of murine $\mathrm{VMC}$, with $\mathrm{CD} 80+\mathrm{B}$ cells being more prominent than $\mathrm{CD} 86+\mathrm{B}$ cells.

\section{Study limitations}

The present study does have limitations. First, the role of CD80+/CD86+ B cells in VMC requires further exploration. Second, prospective studies are essential to further clarify the direct relationship between CD80+ B cells and disease severity.

\section{Acknowledgements}

This work was supported by the National Natural Science Foundation of China (81670345).

The authors declare no conflict of interest.

\section{References}

1. Fung G, Luo H, Qiu Y, et al. (2016): Myocarditis. Circ Res 118: 496-514.

2. Garmaroudi FS, Marchant D, Hendry R, et al. (2015): Coxsackievirus B3 replication and pathogenesis. Future Microbiol 10: 629-653.

3. Liu P, Martino T, Opavsky M A, et al. (1996): Viral myocarditis: balance between viral infection and immune response. Can J Cardiol 12: 935-943.

4. Harris NL, Ronchese F (1999): The role of B7 costimulation in T-cell immunity. Immunol Cell Biol 77: 304-311.

5. Pardigon N, Cambouris C, Bercovici N, et al. (2000): Delayed and separate costimulation in vitro supports the evidence of a transient "excited" state of CD8+ T cells during activation. J Immunol 164: 4493-4499.

6. O'Neill SK, Cao Y, Hamel KM, et al. (2007): Expression of $\mathrm{CD} 80 / 86$ on B cells is essential for autoreactive $\mathrm{T}$ cell activation and the development of arthritis. J Immunol 179: 5109-5116.

7. Podojil JR, Kohm AP, Miller SD (2006): CD4+ T cell expressed CD80 regulates central nervous system effector func- tion and survival during experimental autoimmune encephalomyelitis. J Immunol 77: 2948-2958.

8. Li J G, DU YM, Yan Z D, et al. (2016): CD80 and CD86 knockdown in dendritic cells regulates Th1/Th2 cytokine production in asthmatic mice. Exp Ther Med 11: 878-884.

9. Seko Y, Takahashi N, Yagita H, et al. (1999): Effects of in vivo administration of anti-B7-1/B7-2 monoclonal antibodies on the survival of mice with chronic ongoing myocarditis caused by Coxsackievirus B3. J Pathol 188: 107-112.

10. Seko Y, Takahashi N, Ishiyama S, et al. (1998): Expression of costimulatory molecules B7-1, B7-2, and CD40 in the heart of patients with acute myocarditis and dilated cardiomyopathy. Circulation 97: 637-639.

11. Menezes SM, Decanine D, Brassat D, et al. (2014): CD80+ and CD86+ B cells as biomarkers and possible therapeutic targets in HTLV-1 associated myelopathy/tropical spastic paraparesis and multiple sclerosis. J Neuroinflammation 11: 18.

12. O'Neill SK, Cao Y, Hamel KM, et al. (2007): Expression of CD80/86 on B cells is essential for autoreactive $T$ cell activation and the development of arthritis. J Immunol 179: 5109-5116.

13. De Vooght V, Carlier V, Devos F C, et al. (2013): B-lymphocytes as key players in chemical-induced asthma. PLoS One 8: e83228.

14. O'Neill SK, Cao Y, Hamel KM, et al. (2007): Expression of $\mathrm{CD} 80 / 86$ on $\mathrm{B}$ cells is essential for autoreactive $\mathrm{T}$ cell activation and the development of arthritis. J Immunol 179: 5109-5116.

15. Eriksson U, Ricci R, Hunziker L, et al. (2003): Dendritic cell-induced autoimmune heart failure requires cooperation between adaptive and innate immunity. Nat Med 9: 1484-1490.

16. Chang TT, Jabs C, Sobel RA, et al. (1999): Studies in B7-deficient mice reveal a critical role for B7 costimulation in both induction and effector phases of experimental autoimmune encephalomyelitis. J Exp Med 190: 733-740.

17. Smith-Garvin JE, Koretzky GA, Jordan MS (2009): T cell activation. Annu Rev Immunol 27: 591-619.

18. Brown JA, Greenwald RJ, Scott S, et al. (2002): T helper differentiation in resistant and susceptible B7-deficient mice infected with Leishmania major. Eur J Immunol 32: 1764-1772.

19. Mbow ML, Dekrey GK, Titus RG (2001): Leishmania major induces differential expression of costimulatory molecules on mouse epidermal cells. Eur J Immunol 31: 1400-1409.

20. Furukawa Y, Mandelbrot D A, Libby P, et al. (2000): Association of B7-1 co-stimulation with the development of graft arterial disease. Studies using mice lacking B7-1, B7-2, or B7-1/B7-2. Am J Pathol 157: 473-484.

21. Kuchroo VK, Das MP, Brown JA, et al. (1995): B7-1 and B7-2 costimulatory molecules activate differentially the Th1/ Th2 developmental pathways: application to autoimmune disease therapy. Cell 80: 707-718.

22. O'Neill SK, Cao Y, Hamel KM, et al. (2007): Expression of CD80/86 on B cells is essential for autoreactive T cell activation and the development of arthritis. J Immunol 179: 5109-5116.

23. Nolan A, Weiden M, Kelly A, et al. (2008): CD40 and CD80/86 act synergistically to regulate inflammation and mortality in polymicrobial sepsis. Am J Respir Crit Care Med 177: 301-308.

24. Nolan A, Kobayashi H, Naveed B, et al. (2009): Differential role for CD80 and CD86 in the regulation of the innate immune response in murine polymicrobial sepsis. PLoS One 4: e6600.

25. Leng JH, Hu ZY, Zhuo GC, et al. (2004): [The expression and significance of costimulatory molecule in peripheral blood $\mathrm{B}$ lymphocytes in rheumatoid arthritis]. Zhonghua Nei Ke Za Zhi 43: 519-521. 